These possibilities bear on several lines of thought. Mindlike behaviour in the artefact is seen to be characterized by the circulation of information; and any mode of analysis which makes the latter concept meaningless will exorcise the concept of mind with Procrustean thoroughness. It is suggested that an analogue of the Principle of Complementarity admits of important application here, and that criteria of appropriateness as well as of consistency must be used to test the legitimacy of an analysis.

The artefact is not advanced as a detailed model of the human brain; but the manner in which mentality could be mediated by it, and the nature of its choices, are suggestive. It offers an interpretation of the " $I$ " as the totality of currently-reactive elements organized by information-linkages to constitute an organism ; and the concept of "randomness" here appears in a somewhat new light as the element responsible for the interest and creativeness possessed by the artificial personality so mediated. The analogue of human free will is seen to have a natural place within the framework of physical "law" governing the mechanism.

It is finally suggested that no new theological issues are raised by such developments, though misconceptions of Christian doctrine, both by some Christians and by their opponents, make it important that the " nature of Man " be carefully studied in this new light. To build an artificial man is doubtless impracticable; but the possibility in any case affects in no way the status of choices made by natural men in terms of the calculus of responsibility.

\title{
The Problem of the Existence of Mathematical Entities
}

\section{By Profrssor E. W. BETH}

\section{ABSTRACT of Paper read on 2nd March, 1951.}

The problem of the existence of mathematical entities takes its origin from the fact that, while the truths of mathematics belong to those elements in human knowledge to which we ascribe the highest degree of certainty, we search in vain, in the world of human experience, for entities which present the properties described in these truths. The first attempts to solve it: platonism, aristotelianism, and constructivism (Plotinus, Nicolaus Cusanus, Kepler, Hobbes), belong to speculative philosophy.

The crisis in the foundations of mathematics, however, forced the mathematicians to consider the problem from their own point of view. At first, they borrowed their solutions from speculative philosophy. Later, the development of axiomatics in geometry led Poincaré and Hilbert to accept the formal consistency of a system of axioms as a necessary and sufficient condition for the existence of a model. It was observed by L. Löwenheim (1915) that, for axiom systems formulated within the Peirce-Schröder calculus, the existence of a model can be discussed in a rigorous manner. The development of his ideas led finally to the Löwenheim-Skolem-Gödel theorem : an axiom system formulated within elementary logic has a model, if and only if it is formally consistent. Recently, this result has been extended to various other logical systems by A. Mostowski, L. Henkin, and Helena Rasiowa.

Far from leading to a final settlement of the problem, however, this result was shown by Th. Skolem to give rise to a complete relativisation of mathematical existence. So it seems reasonable to reconsider the problem from the point of view of speculative philosophy ; of course, we cannot expect to obtain, on this basis, a deductive theory of mathematical existence. Nevertheless, it is felt that a realistic and pluralistic conception of human knowledge, which can borrow precious elements from older doctrines, may contribute to a clarification of the situation. 